\title{
Análisis de los hábitos alimenticios con estudiantes de décimo año de un Colegio Técnico en Pérez Zeledón basados en los temas transversales del programa de tercer ciclo de educación general básica de Costa Rica
}

\author{
Analysis of Tenth-Year Students' Eating Habits of a Technical High School in Pérez Zeledón Based \\ on the Transversal Themes of the Program for Third Cycle of Costa Rica Basic General Education
}

Análise dos hábitos alimentares de estudantes de segundo ano do ensino médio de um colégio técnico em Pérez Zeledón, fundamentado nos temas transversais do programa do terceiro ciclo do ensino médio em Costa Rica

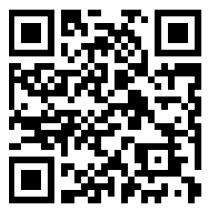
José Miguel Pereira-Chaves ${ }^{1}$
Escuela de Ciencias Biológicas
Universidad Nacional
Heredia, Costa Rica
jose.pereira.chaves@una.cr

María de los Ángeles Salas-Meléndez²

Ministerio de Educación Pública

Pérez Zeledón, Costa Rica

mariucr2005@yahoo.es

Recibido 29 de marzo de 2016 • Corregido 3 de julio de 2017 • Aceptado 20 de agosto de 2017 Received 29 de marzo de 2016 • Revised 3 de julio de 2017 • Accepted 20 de agosto de 2017 Recebido 29 de marzo de 2016 • Revisado 3 de julio de 2017 • Aprovado 20 de agosto de 2017

\footnotetext{
1 Bach en Biología Tropical y Bachiller y Licenciatura en Enseñanza de las Ciencias de la Universidad Nacional de Costa Rica, Maestría en Manejo de Recursos Marinos y Costeros de la Universidad Nacional, Doctorado en Educación de la Universidad Estatal a Distancia. Actualmente es el coordinador de la Maestría en Ciencias Marinas y Costeras, Coordinador de las Olimpiadas Costarricense de Ciencias Biológicas, académico de diversos cursos de la carrera de biología, de la maestría y de Enseñanza de las Ciencias. Ha publicado artículos en distintas revistas indexadas tanto en el campo de biología como del área de enseñanza de las ciencias, así como su participación en diversos congresos relacionados a la enseñanza de las ciencias experimentales.

2 Bachiller en Enseñanza de las Ciencias en la Universidad de Costa Rica y Licenciada en Enseñanza de las Ciencias en la Universidad Nacional de Costa Rica, profesora de Ciencias Experimentales en Educación Secundaria para el Ministerio de Educación Pública de Costa Rica.
} 
doi: http://dx.doi.org/10.15359/ree.21-3.12

URL: http://www.una.ac.cr/educare

CORREO: educare@una.cr

Resumen: El presente trabajo tuvo como objetivo diagnosticar el conocimiento que tiene el estudiantado de décimo nivel sobre hábitos alimenticios y nutrición. Se hace un recuento de la relación entre buena alimentación y los ejes transversales del Programa de Estudio del Tercer Ciclo de Educación General Básica y se considera que la alimentación saludable no debe escapar del ámbito educativo, se deben inculcar los valores y costumbres alimenticias que permitirán al estudiantado desarrollarse de forma óptima en la vida. Se aplicó una encuesta de 13 ítems con alternativas de respuesta cerrada y abierta a 30 estudiantes de décimo año, y a 18 docentes de un Colegio Técnico Profesional de Pérez Zeledón, San José, Costa Rica. Los principales resultados mostraron la poca cultura nutricional que tiene el estudiantado de décimo año en este centro educativo, y el poco compromiso del personal docente para transversalizar en sus clases los hábitos saludables de alimentación.

Palabras claves: Alimentación saludable; nutrición; trastornos alimenticios; ejes transversales; educación.

Abstract: The objective of this study was to diagnose the knowledge tenth-grade students have about eating habits and nutrition. This analysis reviews the relation between good nutrition and the transversal axes of the program of study for the third cycle of Costa Rica's general basic education. Considering that healthy eating should not escape from the educational field, the nutritional values and eating habits should be inculcated to students, so that they can develop optimally in life. A survey containing 13 items with open and closed multiple-choice answers was applied to 30 tenth-grade students, and 18 teachers from a Professional Technical High School located in Pérez Zeledón, San José, Costa Rica. The main results showed the poor nutritional culture of the surveyed students in this educational center, and the lack of commitment of the teachers to mainstream healthy eating habits in their classes.

Keywords: Healthy eating; nutrition; eating disorders; pleasure; transversal axis; education.

Resumo: O objetivo deste estudo foi elaborar um diagnóstico do conhecimento que os alunos de segundo ano do ensino médio possuem sobre hábitos alimentares e nutrição. Para isso, foi feita uma memoria sobre a relação entre a boa nutrição e os temas transversais do Programa de Estudo do Terceiro Ciclo de Ensino Médio e considerou-se que uma alimentação saudável não deve estar separado do campo de educação; deve ser inculcado valores e hábitos alimentares que os estudantes irão aplicar no decorrer de suas vidas. Aplicou-se um questionário com 13 itens com respostas alternativas entre fechadas e abertas a 30 estudantes de segundo ano do ensino médio e a 18 professores de um Colégio Técnico Profissional de Pérez Zeledón, San Jose, Costa Rica Os principais resultados mostraram que os estudantes do segundo ano do ensino médio deste centro educativo tem pouco conhecimento nutricional, e também existe pouco compromisso por parte dos professores em suas aulas para viabilizar os hábitos alimentares saudáveis como um tema transversal.

Palavras-chave: Alimentação saudável; nutrição; distúrbios alimentares; temas transversais; educação. 


\section{Introducción}

En el mundo "más de 900 millones de personas manifiestan desnutrición y aproximadamente 170 millones de niños sufren insuficiencia ponderal" (p. 2), producto de que no reciben los niveles adecuados de nutrientes esenciales para llevar a cabo sus actividades diarias, lo que trae, como consecuencia, deficiencias muy serias en el desarrollo físico y mental del niño o la niña, aspectos que conllevan grandes problemas socioeconómicos a nivel mundial (FAO, 2011).

Pero hablar de nutrición no es simplemente abordar la ausencia de los alimentos, como ocurre en muchos países, sino es referirse a los nutrientes necesarios que el cuerpo requiere para cumplir con todas sus actividades fisiológicas, por ello hablar de alimentación siempre ha sido un tema controversial a lo largo de la historia del ser humano; mientras que para algunos grupos se limita a ser un conflicto entre elegir lo más sabroso de lo menos gustoso, para otros se ha convertido en un determinante entre la vida y la muerte. El ámbito educativo no se escapa de esta problemática, ya que, diariamente, se puede observar un contraste de estudiantes que pueden elegir qué comer y dónde hacerlo, producto de su poder adquisitivo, mientras se tiene gran cantidad de estudiantes para quienes su única comida saludable y nutritiva es la que brinda la institución educativa durante su estadía diaria.

Daza y Arrieta (2010, p. 13) señalan que "los estudiantes, indistintamente emplean los términos alimentación, digestión, ... nutrición, y parece ser originado en la visión compartimentada de los procesos fisiológicos, el desconocimiento de los procesos implicados o la visión deformada y simple de los mismos; de igual forma la palabra alimentos es polisémica y presenta múltiples significados en la cotidianidad de la vida e inclusive en los espacios científicos y está multiplicidad se ve relajada en el conocimiento escolar", que muchas veces no se aborda de manera integral.

La nutrición es un tema que se debe abordar de la mano con la alimentación. Se puede alimentar a una persona, pero no necesariamente se están satisfaciendo las necesidades nutricionales de esta. El estudiantado está expuesto todos los días a un sinfín de recursos alimenticios que atentan contra su salud, sin que estos lleguen a cubrir por completo los lineamientos para obtener placer de forma adecuada.

En Costa Rica, el programa de estudio del 2012 de III ciclo de Educación General Básica, propuesto por el Ministerio de Educación Pública (MEP, 2012), dentro de sus fundamentos teóricos propone, entre otros temas, como eje transversal en los contenidos de Ciencias el de "Educación para la salud". Si bien los ejes transversales no parecen ser los temas más populares para ser trabajados dentro de las lecciones de Ciencias, es necesario hacer esa integración de los términos de salud y placer de forma adecuada. El estudiantado probablemente no perciba un gran nivel de afectación en su salud, debido a su edad, sin embargo, en los años posteriores es posible se vea las consecuencias de no tener una formación sólida en cuanto a buenos hábitos 
doi: http://dx.doi.org/10.15359/ree.21-3.12

URL: http://www.una.ac.cr/educare

CORREO: educare@una.cr

alimenticios. Esto se evidencia aún más con la tendencia a la obesidad que se ha venido dando en la niñez y juventud desde hace algunos años y que comúnmente se aborda en los diferentes medios de comunicación masiva.

Es necesario dejar claro que cuando se habla de hábitos alimenticios se debe observar el panorama completo que conduce al estudiantado a ingerir una determinada comida. Probablemente este se encuentre bombardeado por una enorme cantidad de publicidad que lo induce a comprar comidas que no son las que realmente el cuerpo le agradecerá a mediano y largo plazo. También puede que la publicidad misma impida al estudiantado considerar la existencia e importancia de una alimentación digna de su salud. Además, el contexto cultural enmarca muchas de las afinidades juveniles hacia los alimentos. Gran parte de la cultura costarricense se basa en el consumo de carbohidratos en su gran mayoría desde las diversas formas de ingestión.

El objetivo de este trabajo fue analizar los hábitos alimenticios que tiene el estudiantado del Tercer Ciclo de Educación General Básica en un Colegio Técnico de la Regional de Educación de Pérez Zeledón, con el fin de diagnosticar la realidad sobre los hábitos alimenticios que posee en cuanto a lo que consumen diariamente.

\section{Referente teórico}

\section{La alimentación, hábitos alimenticios y nutrición}

La alimentación es el ingreso o aporte de los alimentos en el organismo. Es el proceso por el cual se toma una serie de sustancias contenidas en los alimentos que componen la dieta. Estas sustancia o alimentos son importantes para tener una adecuada nutrición (Fernández, 2003). Así, la alimentación es una necesidad fisiológica para el organismo, y se constituye una importante dimensión social y cultural, vinculada por un lado a saciar el hambre y por otro al buen gusto, y la combinación de ambos factores puede llegar a generar placer. Es fundamental resaltar que durante el acto de comer se activan los sentidos como la vista, olfato, gusto y tacto y, por último, el oído puede intervenir al recibir mensajes publicitarios sobre alimentos.

Se tienen diversos abordajes en la definición de hábitos alimenticios, debido a que existe una diversidad de conceptos, pero "la mayoría converge en que se trata de manifestaciones recurrentes de comportamiento individuales y colectivas respecto al qué, cuándo, cómo, con qué, para qué se come y quién consume los alimentos, y que se adoptan de manera directa e indirectamente como parte de las prácticas socioculturales" (Moreiras y Cuadrado, 2001; Ortiz, Vázquez y Montes, 2005, citados por Macias, Gordillo y Camacho (2012, p. 41).

Desde la experiencia de quienes escriben el artículo, se define hábito alimenticio como las acciones que conllevan la selección de comida para satisfacer las necesidades fisiológicas del cuerpo, por lo que la ingesta de alimentos de una población está muy relacionada con sus costumbres, tradiciones, contexto socioeconómico y familiar.

4 
Albito (2015) señala:

Los hábitos alimentariosforman parte de cada persona, pero no nacen con él se forman a partir de experiencias del aprendizaje adquiridas a lo largo de la vida, mediante cual el individuo selecciona los alimentos que han de configurar su dieta y los prepara para su ingestión. Por tanto, es un proceso voluntario y educable que constituye un factor determinante en su estado de salud. Estos hábitos pueden ser inadecuados por déficit o exceso y se relacionan con numerosas enfermedades como: sobrepeso, obesidad, anemia etc. (p. 4)

En los hábitos alimentarios intervienen tres factores principalmente, por ejemplo, si se aborda desde la génesis del individuo, comienza en el núcleo familiar, donde este influye, en gran medida, a que niños, niñas y adolescentes adquieran conciencia de una buena alimentación; por otra parte se tiene la escuela o centro educativo, en donde se pueden reforzarse en el medio escolar los buenos hábitos alimenticios a través de la transversalidad del contenido. En Costa Rica se ha normado la venta de alimentos en los centros educativos para prevenir la ingesta poco nutritiva de estos y así controlar en el interior de la escuela que el alumnado no coma los llamados alimentos "chatarra"; por otra parte, el tercer influyente en la ingesta de alimentos son los medios de comunicación masiva en la publicidad ejercida por las empresas agroalimentarias, en ocasiones, invasivas y manipuladoras. Por ello, las conductas observables en las personas adultas muchas veces son reproducidas en las generaciones de menores, pues, en gran parte, la alimentación se deriva de una tradición étnica establecida históricamente entre las diferentes culturas alimentarias.

El incentivar los buenos hábitos alimentarios y promover en el estudiantado el análisis sobre las posibles consecuencias de ello, es una excelente oportunidad para prevenir enfermedades y promover la salud en la población. La mejor manera de alcanzar un estado nutricional adecuado es incorporar una amplia variedad de alimentos a nuestra dieta diaria y semanal. Por ejemplo, la FAO (2011) señala que "cientos de millones de personas padecen de enfermedades derivadas de regímenes alimenticios excesivos o poco equilibrados, y muchos países en desarrollo hacen actualmente frente a graves problemas de salud en los dos extremos del espectro nutricional" (p. 2).

Son muchos los factores que intervienen en la buena nutrición, por ejemplo, González y Merino (2007) señalan:

Hoy día, la disponibilidad de alimentos elaborados, las redes de distribución de los productos frescos, el precio de los alimentos, ponen a disposición de todas las mesas un número de productos alimentarios enorme, muchas veces ya elaborados, que complican enormemente la selección y la combinación saludable de los alimentos.

Si a esto unimos que nuestra sociedad se ha mecanizado por completo, tenemos como resultado una nueva dificultad para mantenernos sanos. (p.7) 
doi: http://dx.doi.org/10.15359/ree.21-3.12

URL: http://www.una.ac.cr/educare

CORREO: educare@una.cr

Uno de los pilares fundamentales de la salud, del adecuado crecimiento y desarrollo es la nutrición, la cual se realiza en cada una de los millones de células del cuerpo humano, y hay que considerar que el metabolismo propio de cada individuo influye directamente en ello, por eso las reacciones metabólicas que se generan en el organismo están fuertemente determinadas por el tipo de alimentación de cada persona, por lo que la elección de alimentos, la forma de preparación y consumo, es la base de una adecuada nutrición. Por otra parte, Macias et al., 2012, señalan que:

Considerando que la alimentación y la nutrición son procesos influenciados por aspectos biológicos, ambientales y socioculturales y que durante la infancia contribuyen a un desarrollo y crecimiento óptimo, así como una maduración biopsicosocial, es necesario que los niños adquieran durante esta etapa hábitos alimentarios saludables. Sin embargo, para ello es necesario considerar factores de tipo fisiológicos, sociales y familiares, donde estos últimos ejercen una fuerte influencia en los patrones de consumo. No obstante, los hábitos alimentarios se han ido modificando por factores que alteran la dinámica familiar tales como la menor dedicación y falta de tiempo para cocinar y la pérdida de autoridad en cuanto a la cantidad y calidad de los alimentos que consumen los niños. (p. 40)

Por lo que se debe tener un abordaje integral de la nutrición, especialmente en etapas tan fluctuantes y de grandes cambios hormonales como la adolescencia, por ello se destaca lo citado por Marugán, Monasterio y Pavón (2010):

La alimentación del adolescente debe favorecer un adecuado crecimiento y desarrollo y promover hábitos de vida saludables para prevenir trastornos nutricionales. Esta etapa puede ser la última oportunidad de preparar nutricionalmente al joven para una vida adulta más sana.

Pero, por otra parte, en esta época pueden adquirirse nuevos hábitos de consumo de alimentos, debido a varios factores: influencias psicológicas y sociales, de los amigos y compañeros, el hábito de comer fuera de casa, el rechazo a las normas tradicionales familiares, la búsqueda de autonomía y un mayor poder adquisitivo. (p. 307)

La pubertad y la adolescencia se caracterizan por importantes modificaciones en el organismo, se da un aumento de la velocidad de crecimiento, cambios en la composición corporal, aparición de caracteres sexuales secundarios y maduración emocional y psicosocial, varían su morfología y composición. Los requerimientos nutricionales dependen del gasto necesario para mantener el ritmo de crecimiento, de las variaciones en la composición corporal y del consumo energético. La nutrición correcta es una de las necesidades básicas de salud para que la población adolescente pueda desarrollar, al máximo, su potencial biológico (Aguirre, Castillo y Le Roy, 2010). 
Un problema de salud que se encuentra en los centros educativos y que se relaciona con las prácticas alimentarias son la anorexia y la bulimia. La RAE (2014) define la anorexia como "síndrome de rechazo de la alimentación por un estado mental de miedo a engordar, que puede tener graves consecuencias patológicas" y la bulimia como un "síndrome de deseo compulsivo de comer, con provocación de vómitos y consecuencias patológicas". Por esto, la educación nutricional debe dirigir sus campañas, mediante técnicas y métodos eficaces, a rescatar a la población de las malas prácticas alimentarias y contribuir a la reducción de enfermedades.

\section{Factores de riesgo nutricional}

La autonomía e independencia propias de la edad se acompañan de un incremento de las actividades que adolescentes llevan a cabo fuera de su hogar, incumpliendo los horarios de ingesta de alimentos, no consideran los tiempos de comida como importantes y, por eso, en muchas ocasiones evaden la responsabilidad de su propia alimentación. En la actualidad hay un incremento en la ingesta de comida rápida, golosinas y bebidas energéticas durante las comidas y, por otro lado, se disminuye el consumo de productos lácteos, frutas, verduras, legumbres Este aspecto, unido al sedentarismo resulta muy preocupantes debido a que ha venido contribuyendo a los riesgos de obesidad y otras enfermedades crónicas (Neimeier, Raynor, Lloyd-Richardson, Rogers y Wing, 2006).

Hay evidencia sobre la importancia que se le debe a los aspectos nutricionales en jóvenes. Al respecto, Lujan (2013) señala:

La nutrición está pasando al primer plano como un determinante importante de enfermedades crónicas que puede ser modificado, y no cesa de crecer la evidencia científica en apoyo del criterio de que el tipo de dieta tiene una gran influencia, tanto positiva como negativa, en la salud a lo largo de la vida. Lo que es más importante, los ajustes alimentarios no sólo influyen en la salud del momento, sino que pueden determinar que un individuo padezca o no enfermedades tales como cáncer, enfermedades cardiovasculares, hipertensión, osteoporosis, dislipemia y diabetes. (pp. 29-30)

Otro hábito adolescente poco saludable es incluir otros alimentos a su dieta alimenticia como la llamada comida chatarra, dulces, y las bebidas gaseosas. Esta comida chatarra acarrea consigo que suban de peso, tengan problemas de piel, y no rindan físicamente ni a nivel escolar, ya que son alimentos con alto contenido en grasa.

Existen otras situaciones que también constituyen un factor de riesgo nutricional como la enfermedad crónica, embarazo, actividad deportiva, medicación crónica y abuso de drogas. El inicio temprano en el consumo de alcohol (en forma aguda o crónica) y el tabaquismo tiene 
doi: http://dx.doi.org/10.15359/ree.21-3.12

URL: http://www.una.ac.cr/educare

CORREO: educare@una.cr

repercusiones sobre el desarrollo de las habilidades sociales e interpersonales, ya que afecta un sistema nervioso inmaduro. Generalmente, el abuso en la ingestión de alcohol y el tabaquismo no es una conducta que se dé en forma aislada; por lo general, se presenta en jóvenes que tienen problemas de rendimiento escolar, pertenecen a hogares desintegrados y son candidatos o candidatas a consumir otras sustancias prohibidas.

\section{Problema nutricional del sujeto adolescente}

En general, la mayor preocupación de los diversos países son los altos índices de obesidad que tiene su población y en espacial en la niñez y adolescencia. Flegal, Carroll, Ogden y Johnson (2002), en la Encuesta Nacional de Salud y Nutrición en Estados Unidos, señalaron que el 10\% de la población entre 2 y 5 años, y $15 \%$ entre 6 y 19 años tienen sobrepeso en este país. La prevalencia de sobrepeso en la población infantil y adolescente se ha duplicado en los últimos veinte años y triplicado en los últimos cuarenta. Existen otros estudios que demuestran que entre 20 y 40 por ciento de jóvenes estadounidenses presentan problemas físicos. Solo un pequeño porcentaje de niñez obesa puede atribuir su problema a trastornos endócrinos u otros problemas físicos similares.

Lamentablemente, Costa Rica no escapa a esta realidad, ya que actualmente la obesidad es el trastorno nutricional más prevalente en niños, niñas y adolescentes. Serrú, Laclé y Coto (2003), nos dicen, al respecto:

En la última encuesta Nacional de Nutrición la prevalencia de obesidad en mujeres de 15 a 19 años fue de un $23.2 \%$ y en escolares en la zona metropolitana del $20 \%$. El grado de sobrepaso o riesgo de obesidad se diagnostica con el indicador IMC; en poblaciones con porcentajes importantes de personas "achicadas", como es el caso de los países en desarrollo, como [Costa Rica].... Considerando las tendencias de obesidad en nuestro país, es necesario conocer la magnitud de este problema en nuestra población adolescente ya que muchos estudios determinan que ésta prevalece hasta la edad adulta y [que] a su vez es un factor de riesgo para muchas enfermedades crónicas. (p. 53)

El Programa de Estudio de Ciencias del III Ciclo del MEP (2012), para noveno nivel, contiene el tema de la salud y nutrición, cuyo objetivo es reconocer la organización de las células en organismos pluricelulares y valorar las funciones que cumplen los tejidos, órganos y sistemas, así como la necesidad de contribuir a su mantenimiento y a la prevención de acciones nocivas para la salud.

El tema nutrición ehigiene, base de la salud, contenido de este programa, debe desarrollarse en un tiempo de tres lecciones, por consiguiente no es mucho lo que se puede profundizar; por lo tanto, es muy probable que los conocimientos que obtiene el estudiantado y la intervención 
docente en este lapso no provoquen la reflexión y las competencias del pensamiento científico como lo es el analizar, compartir, argumentar y, sobre todo, debatir sobre la importancia de ingerir alimentos aptos para la obtención de una buena nutrición.

La incorporación de los temas transversales es una de las últimas disposiciones realizadas en el ámbito educativo de nuestro país. El Ministerio de Educación Pública de Costa Rica ha incluido directamente los temas transversales al currículo escolar. Corresponde al personal docente, mediante su labor educativa, la ejecución e implementación en el aula, con el fin de contribuir a la socialización, promoción y práctica de los valores en el ambiente escolar.

En Costa Rica, los temas transversales que se han visto como prioritarios son los siguientes: Cultura ambiental para el desarrollo sostenible.

- Educación para la salud.

- Educación integral de la sexualidad.

Vivencia de los derechos humanos para la democracia y la paz. (Segura, 2006, p. 2)

La transversalidad nace debido a que existe en el sistema educativo costarricense "una dificultad ... de incorporar nuevas asignaturas o contenidos relacionados con los temas emergentes de relevancia para la sociedad, [ya que] se corre el riesgo de saturar y fragmentar los programas de estudio" (Méndez, 2012, p. 3).

La Comisión de Temas Transversales señala que la salud es un derecho fundamental de todos los niños, niñas y adolescentes, ya que el estado de salud está relacionado con su rendimiento escolary con la calidad de vida. Al trabajar en educación para la salud, en los centros educativos, se está formando una ciudadanía con estilos de vida saludables que construyen una buena calidad de vida para sí y para quienes les rodean.

Aunque todos los temas transversales tienen relación directa o indirectamente con la salud de la población, el eje transversal de educación para la salud es el que se trabaja en esta investigación y en su abordaje se deben inculcar, en el estudiantado, las competencias del pensamiento científico para brindarle las herramientas para la vida y así que pueda dar respuestas a situaciones problemáticas, como los hábitos alimenticios saludables. CalañasContinente (2005) señala que "la alimentación no saludable y el sedentarismo pueden ser responsables de una proporción considerable de fallecimientos evitables, una modificación de éstos podría repercutir notablemente sobre la salud pública" (p. 8). Por ello se deben promover en los salones de clases hábitos alimenticios saludables. 
doi: http://dx.doi.org/10.15359/ree.21-3.12

URL: http://www.una.ac.cr/educare

CORREO: educare@una.cr

\section{Propuesta metodológica}

Esta investigación se basó en un diseño no experimental, transeccional descriptivo mediante un estudio etnográfico, el cual pretende describir y analizar ideas, creencias, significados, conocimientos y prácticas de grupos, culturas y comunidades (Patton, 2002, citado por Hernández, Fernández y Sampieri, 2010).

La investigación se llevó a cabo durante el primer semestre del 2014 en el cantón de Pérez Zeledón, de la provincia de San José. Por su parte, se seleccionó como muestra a todo el estudiantado conformado por 30 discentes cuyas edades oscilan entre los 15 y 18 años y a 18 discentes de la institución, lo que permitió describir las actitudes y opiniones sobre los hábitos alimenticios. considerando que los temas transversales tienen que ser brindados por todo el personal docente, indistintamente de la materia que imparta y su especialidad. La mayoría de la población estudiantil proviene de zonas aledañas las cuales son rurales.

Los criterios de selección de estudiantes de décimo año se deben a que los estudiantes y las estudiantes de décimo grado eran los que habían recibido los contenidos correspondientes a salud y nutrición, y además se consideró que el ciclo diversificado los temas transversales propuestos en esta investigación deben haber sido abordados, por lo que la muestra fue intencional. Hernández et al. (2010) señalan que en el muestreo intencional quien investiga puede elegir ciertos casos, analizarlos, y más adelante seleccionar casos adicionales para confirmar o no los primeros resultados. Posteriormente puede elegir casos homogéneos y luego heterogéneos para probar los límites y alcances de sus resultados.

Para determinar los conocimientos del estudiantado sobre hábitos alimenticios y nutrición adquiridos se aplicó una encuesta que contenía 13 ítems, los cuales fueron construidos con base en preguntas que describiera el uso del comedor, la educación recibida, alimentos nutricionales, identificación de la diferencia entre alimento y nutrición, actividades del centro educativo que promuevan buenos hábitos alimenticios, aportes del profesorado sobre hábitos alimenticios, opinión de estudiantes sobre la importancia de los hábitos alimenticios, que se respondía con un sí o no; además, se hace una pregunta sobre en que año comenzó a recibir información sobre los hábitos alimenticios y preguntas abiertas sobre lo que más le llamó la atención sobre los hábitos alimenticios.

Por otra parte, para determinar en el estudiantado los hábitos alimenticios se aplicó una encuesta que contenía 16 ítems con alternativas de respuesta cerrada: Siempre, Algunas veces y Nunca. Las preguntas fueron elaboradas con base en los objetivos planteados como las siguientes: desayunan todos los días, consumen comida rápida, acostumbras comer golosinas entre comidas, toman al menos seis vasos de agua, consumen frutas, revisan etiquetas nutricionales, toman gaseosas, tienen sobrepeso, realizan dieta para adelgazar, practican deportes, consumen medicamentos para gastritis. 
Para analizar la disposición del personal docente referente a la educación para la salud y su incorporación como materia transversal, se aplicó una encuesta a 18 docentes de la institución, que contenía 16 ítems con alternativas de respuesta abiertas y cerradas cuyas preguntas contenían párrafos sobre los ejes transversales, además de preguntas como: contempla en el centro educativo la educación para la salud, la educación específica que tiene sobre el tema transversal hábitos alimenticios y salud, el tipo de educación que se imparte, causas de los malos hábitos alimenticios en el centro educativo, se aborda el tema transversal en su colegio, metodología empleadas para el abordaje de los temas transversales, disposición de ayudar a una propuesta metodológica.

Posterior a la elaboración de los diferentes cuestionarios de recolección de datos, estos fueron validados por juicio de personas expertas, con tres especialistas en el tema. "Los expertos son personas cuya especialización, experiencia profesional, académica o investigativa relacionada al tema de investigación, les permite valorar, de contenido y de forma, cada uno de los ítems incluidos en la herramienta" (Soriano, 2014, p. 25). Se consideraron los siguientes elementos: a) pertinencia del contenido de los enunciados, b) contextualización de las preguntas a la población meta, c) claridad de las preguntas, d) relación con la teoría y e) coherencia con los objetivos de investigación y sus categorías de análisis. y también se les aplicaron a 10 estudiantes de la carrera de Enseñanza de las Ciencias de la Universidad Nacional sede Omar Dengo, para verificar si había alguna palabra o texto de complejidad para un estudiante.

\section{Resultados y discusión}

Con base en los resultados obtenidos se evidenció que el $57 \%$ del estudiantado ha recibido el tema hábitos alimenticios y nutrición, mientras que un $43 \%$ expresaron no haberla recibido.

Sin embargo, considerando que es un tema transversal en donde todos los docentes de las distintas materias deben abordarlo, según lo demanda la propuesta del plan de estudio, se obtuvo un alto porcentaje casi el $50 \%$ que señala no haber recibido contenido sobre hábitos alimenticios a pesar de que existen tres docentes de ciencias que dan III ciclo, por lo que se podría argumentar que el tema no se está abordando de una manera adecuada, aunque esté incluido en el programa de estudio. Una de las posibles razones de que no se aborde el tema transversal podría ser que ningún profesor del centro educativo integra el mismo en sus materias disciplinares como eje transversal, o a que el aprendizaje no fue significativo para el discente puesto que no lo recuerda. Aquí lo esencial es que pueda darse un trabajo colectivo y coordinado con la institución para que se aborde de manera integral la temática. Hay que considerar que hay estudios como el de Castañeda-Sanchez, Rocha-Díaz y Ramos-Aispuro (2008) quienes señalaron que en la mitad de la población estudiada los hábitos fueron malos, posiblemente por la ingesta de alimentos rápidos o comida chatarra, favorecida por las actividades a que se ven sometidos los adolescentes. 
doi: http://dx.doi.org/10.15359/ree.21-3.12

URL: http://www.una.ac.cr/educare

CORREO: educare@una.cr

Es importante señalar que en la acción educativa se trata en definitiva de orientar la tarea docente como un trabajo colectivo de innovación, investigación y formación permanente, por lo que se hace necesario la reflexión y la autocrítica. Por ello el personal docente debe de mostrar predisposición al cambio, al trabajo en equipo, al planeamiento conjunto y a una flexibilidad curricular donde sea esta temática el trabajo grupal de un proyecto curricular donde se ponga en escena la propuesta de los temas transversales como contenido a desarrollar, considerando que más allá de abordar o articular un contenido se deben optimizar los esfuerzos en el desarrollo de conductas permanentes y en la adquisición de herramientas válidas, además de potenciar la habilidades de las competencias del pensamiento científico para que el estudiantado pueda desenvolverse ante los problemas que se presenten en la vida cotidiana.

La Figura 1 muestra que la mayoría de estudiantes aseguró haber abordado el tema en noveno nivel, que es donde el programa de ciencias lo contiene, aunque se encuentra dentro de los temas transversales para todo el tercer ciclo.

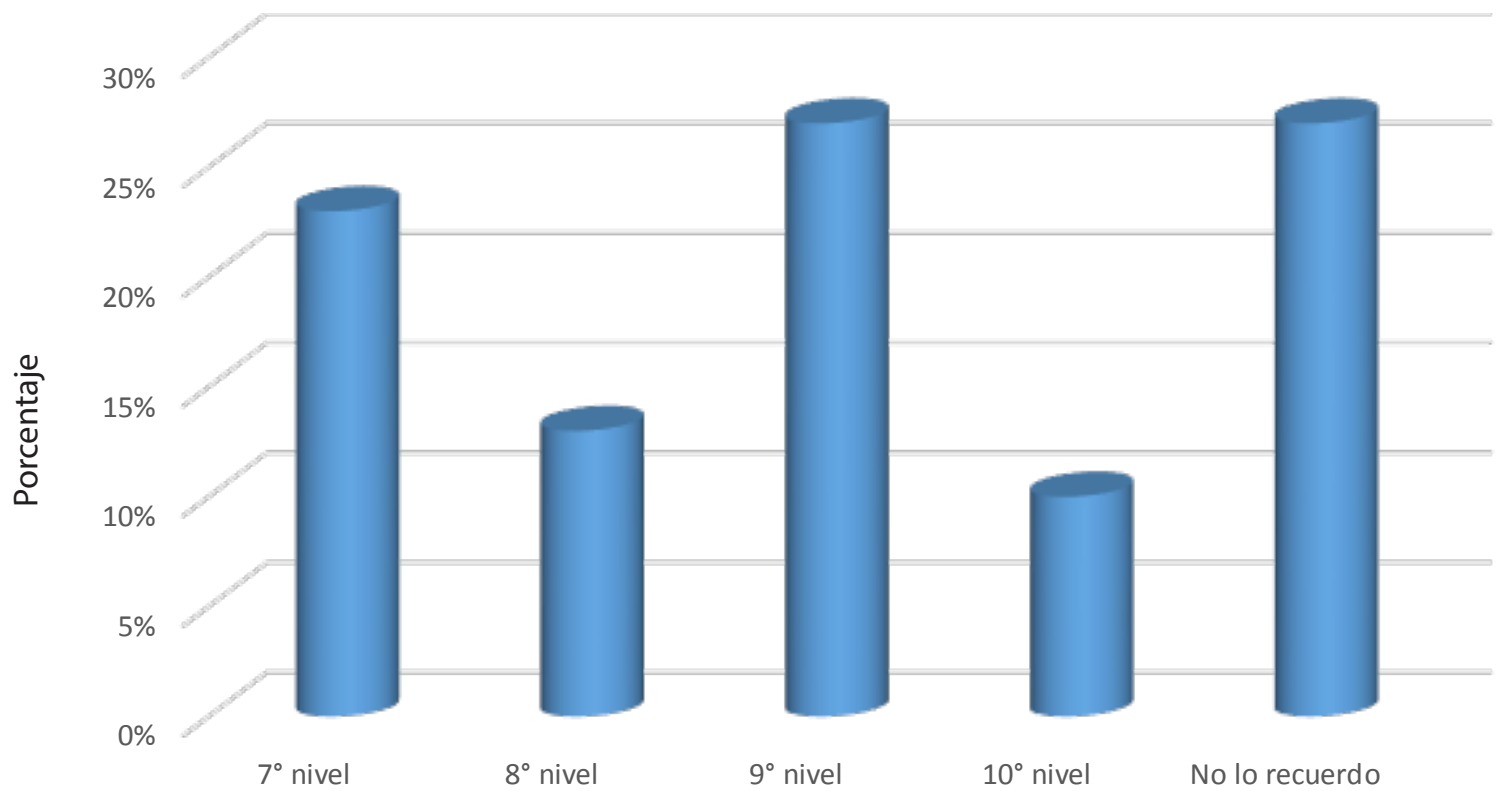

Nivel del estudiantado

Figura 1: Datos obtenidos del estudiantado de décimo nivel sobre el año en que se abordó el tema de Hábitos Alimenticios. 
Es importante señalar que el personal docente debe tener una mayor integración disciplinar y por ello la formación profesional debe darles una adecuada preparación y así las herramientas metodológicas que les permitan interconectar los contenidos con otras disciplinas, además de poner elementos comunes de intercambio, de formación entre los diversos niveles del currículo escolar y poder ir elaborando un proceso de transversalidad en los diferentes años escolares donde se forme integralmente al estudiantes.

Palos (2001) señala que

el acto por parte del profesorado de transversalizar ... [los] contenidos disciplinares con otros, que considera que no están presentes y que son fundamentales en la educación de sus alumnos, siempre ha sido un indicador de que el currículo y lo que se enseña en la escuela no recogen suficientemente la realidad social en la que están inmersos, ... esta transversalización con otros contenidos se ha hecho a título personal y fundamentalmente en algunos de los temas de la materia impartida por el profesorado, pero no se ha hecho de forma globalizada en todo el currículo escolar. (p. 37).

Por otra parte, Díaz y Ramos, 2007) señalan:

Proyectos transversales de carácter curricular constituyen una alternativa eficaz para lograr una coherencia interna entre las diferentes materias que se enseñan, sin embargo, el acontecer del proceso formativo adolece de una falta de interconexión disciplinar en los niveles del año académico y la disciplina, lo que ocasiona un enclaustramiento de las materias impartidas ... (p. 2)

Además, es importante formar al estudiantado no solo en contenidos, sino también potenciar habilidades, destrezas, capacidades científicas, argumentativas que les formen como seres humanos integrales que puedan vivir en armonía con la naturaleza y tengan una mejor calidad de vida, que se puede realizar a través de los valores y los ejes transversales del currículo.

La Figura 2 muestra la opinión del estudiantado en cuanto a si existe relación entre lo que comen y su salud.

La Figura 2 muestra que la mayoría del estudiantado no encontró una relación significativa entreloquecomeny su salud. Uno delosaspectosque puedeestar influyendoesel desconocimiento por parte de los jóvenes entrevistados sobre la manera en que influye la alimentación en la salud de las personas y por ende las consecuencias futuras de algunos trastornos alimenticios que podría presentar. Por otra parte, Montero, Úbeda y García (2006, p. 467) citando a López (2002) quien señala que hay varios estudios epidemiológicos donde "muestran que aunque en ocasiones la población está informada y conoce los conceptos básicos de una dieta saludable, estos conocimientos no se traducen en consumos reales de alimentos que formen parte de una dieta equilibrada y [éstos] no se ponen en la práctica los conceptos aprendidos". 
doi: http://dx.doi.org/10.15359/ree.21-3.12

URL: http://www.una.ac.cr/educare

CORREO: educare@una.cr

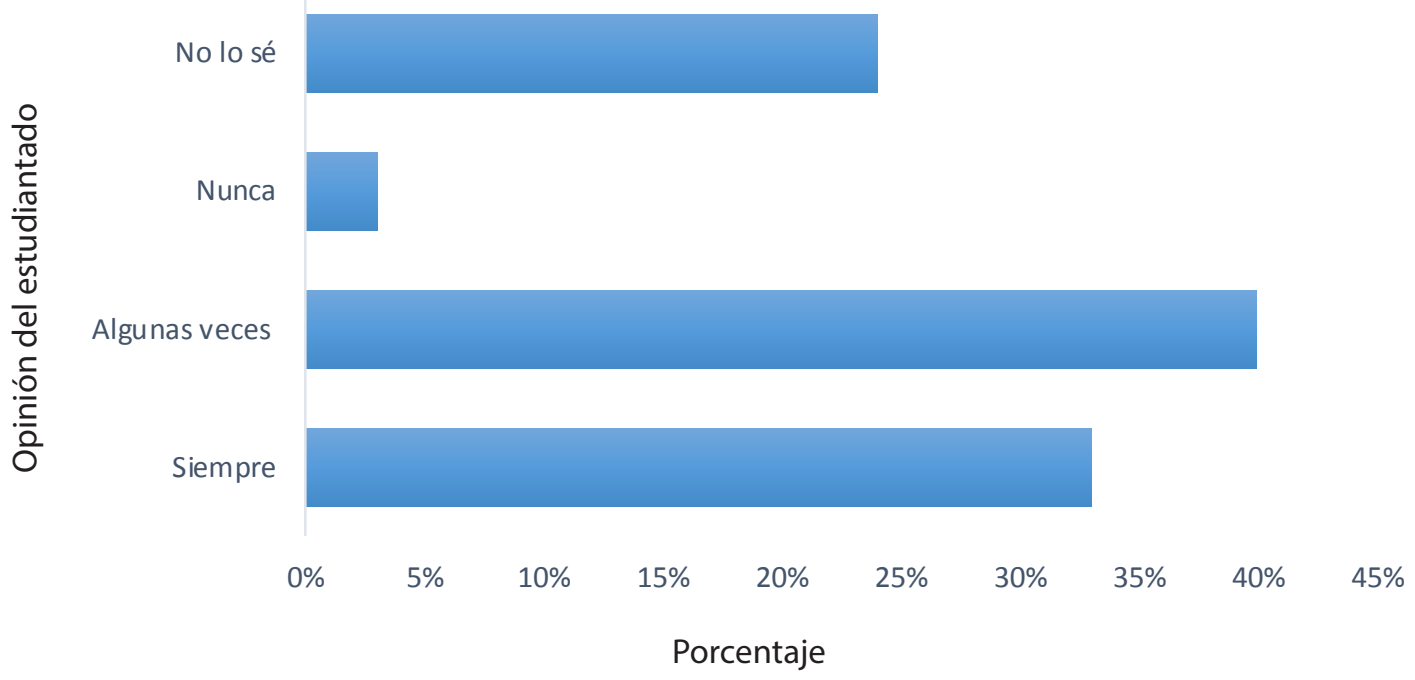

Figura 2: Opinión del estudiantado de décimo nivel sobre la relación entre los hábitos alimenticios y las consecuencias para la salud.

Según Vilaplana (2011), Una dieta es equilibrada cuando el "aporte de los distintos nutrientes respete las proporciones adecuadas indispensable para un óptimo crecimiento y para el mantenimiento de la salud [del individuo]" (p. 46).

Es importante recalcar que los alimentos contienen nutrientes que cumplen funciones específicas para que nuestro organismo crezca, se desarrolle y trabaje adecuadamente. Según Restrepo, Morales, Ramírez, López y Varela (2006), el descubrimiento de los nutrientes y las funciones que desempeñan dentro denuestro organismo nos hapermitido conocer perfectamente muchas de las propiedades de los alimentos que hasta hace relativamente pocos años se intuían o formaban parte de la sabiduría popular. Los avances científicos nos introducen a fondo en el mundo de la alimentación y la relación que los hábitos alimentarios mantienen con la salud.

La Tabla 1 muestra los datos obtenidos sobre los hábitos de los discentes en cuanto a pasar periodos largos sin ingerir alimentos, mayores de seis horas.

La Tabla 1 muestra que, de los 30 estudiantes entrevistados, un 93\% manifestaron que pasan lapsos mayores de seis horas sin ingerir alimentos. Es necesario brindar a los docentes además de los conocimientos sobre los temas de salud y nutrición, lo importante que es no pasar lapsos de tiempos tan largo sin ingerir alimentos, lo cual también provoca un trastorno alimenticio debido a las actividades fisiológicas propias del cuerpo. 
Tabla 1: Frecuencia de los alumnos de décimo nivel de un Colegio Técnico Profesional de la región educativo de Pérez Zeledón con respecto a los periodos de ayuno superiores a seis horas

\begin{tabular}{lc}
\hline Alternativas de respuesta & Total \\
\hline Siempre & 7 \\
Algunas veces & 21 \\
Nunca & 2 \\
\hline Total & 30 \\
\hline
\end{tabular}

Nota: Elaboración propia.

Cuando las personas pasan largos periodos de ayuno tienden a consumir grandes cantidades de calorías cuando pueden volver a comer, lo que ocasiona un aumento de peso y este, a su vez, traerá consecuencias nocivas a su salud. Así se vislumbra una carencia en la educación para la salud que posee este estudiantado. Educación para la salud según Gómez (2004), "constituye la suma de todas aquellas experiencias de un individuo que modifican su comportamiento con respecto a la salud y los procesos y esfuerzos para producir estas modificaciones" (p. 5).

Las personas al ayunar dejan al organismo sin recibir nutrientes, siendo perjudicial para la salud, en esta misma línea Albero, Sanz y Playán (2004, p. 18), señalan, que "es básico asegurar el aporte suficiente de energía a los órganos vitales, preferentemente el cerebro y, a su vez, moderar la pérdida demasiado rápida de las estructuras corporales que sirven como fuente de los productos energéticos". Por ello se hace necesario el espacio para evaluar los hábitos nutricionales, conservar así lo saludable y modificar lo poco saludable para desarrollar una vida sana.

Otra de las interrogantes planteadas al estudiantado fue la frecuencia con que estos ingieren comida chatarra. Para esta pregunta, el 100\% argumentó comer algunas veces por semana este tipo de comida, a pesar de tener conocimiento sobre el exceso de calorías y grasas que contienen estos alimentos. Es válido resaltar que pese a las acciones que el Gobierno ha hecho (a través del Ministerio de educación Pública y la Caja Costarricense del Seguro Social) para disminuir el consumo de comida chatarra en la población estudiantil, este esfuerzo no ha producido los frutos deseados; podría ser que los jóvenes están influenciados por una serie de factores tales como los anuncios publicitarios dirigidos por la empresas fabricantes o distribuidoras de estos productos, lo que nos alerta a que se debe fortalecer el papel de la educación en este campo, para contrarrestar los efectos de este tipo de propaganda. 
doi: http://dx.doi.org/10.15359/ree.21-3.12

URL: http://www.una.ac.cr/educare

CORREO: educare@una.cr

Según French, Story, Fulkerson y Gerlach (2003), las prácticas relacionadas con la alimentación de los adolescentes han sufrido cambios en los últimos tiempos, con una tendencia hacia lo no saludable, que consiste en el incremento del consumo de gaseosas, meriendas, comidas rápidas y azúcares. El poseer una dieta poco saludable trae repercusiones en la salud de las personas sobre todo en etapas de desarrollo.

Cuando se habla de los productos alimenticios al alcance de los estudiantes hay que hacer mención también a las golosinas. Cuando se le preguntó al estudiantado la frecuencia con que consumen golosinas (dulces) se obtuvo que, el $97 \%$ consumen por lo menos algunas veces por semana golosinas entre las comidas, mientras que solamente un $3 \%$ asegura no tener esta práctica.

El principal ingrediente de las golosinas es el azúcar, y el exceso de azúcar en el organismo genera que éstas se acumulen en forma de grasa, así como también que se vaya a la sangre y pueda producir enfermedades como la diabetes y afecciones propias del sistema nervioso. Otra consecuencia del consumo de golosinas son las caries, estas contienen alimentos para las bacterias que se producen en la boca y que son causantes de caries, mal aliento entre otros.

Las golosinas constituyen un exceso de calorías con poco valor nutricional que en algunos casos podrían volverse adictivos provocando sobrepeso y afectando la salud en general. Según Castillo y Romo (2006,"Impacto de las golosinas", párr. 3), "las golosinas incrementan la densidad energética y la proporción de energía proveniente de la grasa e hidratos de carbono, mientras que son deficientes en otros nutrientes", por lo que se debe seguir insistiendo en la promoción de la educación para la salud para modificar las conductas individuales y colectivas de este y todos los grupos de la población.

En este aspecto la gestión escolar y las autoridades del MEP representan un apoyo para contribuir al no consumo de dulces dentro de los centros educativos eliminándolos por completo de las sodas escolares. Y a su vez los padres de familia en primer lugar y los educadores como apoyo inculcar en los educandos buenos hábitos alimenticios.

La Figura 3 muestra el porcentaje de estudiantes que leen las etiquetas con la información nutricional de los alimentos que consumen.

En Figura 3 se puede observar que la mayoría de discentes entrevistados anotaron que algunas veces o nunca leen las etiquetas con la información nutricional que traen los alimentos. Es importante conocer la información nutricional de los alimentos que consumimos para así seleccionar los más adecuados. Sedó (2009), señaló que el consumidor de alimentos preocupado por su salud, la meta es comprar alimentos nutritivos, ricos y de bajo costo, y una adecuada forma de hacerlo es revisando la etiqueta nutricional de los alimentos la que indica el valor energético y el contenido del alimento que se va a consumir. 


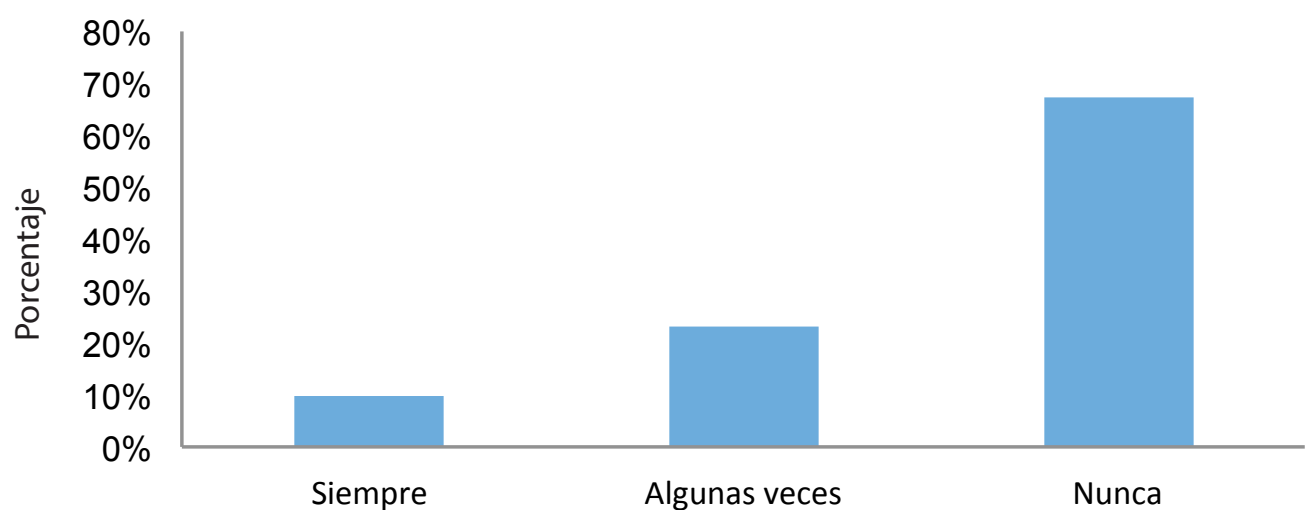

Frecuencia de lectura

Figura 3: Criterio obtenido del estudiantado sobre la lectura de etiquetas con información nutricional de los alimentos.

Ante la carencia de una conciencia reflexiva y responsable de la población sobre los hábitos de nutrición y estilos de vida saludables, nace la necesidad de apoyar la labor pedagógica con estrategias que vengan a fortalecer estas y otras áreas débiles del ámbito escolar y cotidiano. El Ministerio de Educación Pública ha puesto al alcance de los docentes el eje y los temas transversales como instrumento didáctico sustentado en valores y competencias para orientar y reforzar las prácticas educativas.

El papel de la educación es fundamental en este punto para desarrollar en la juventud la cultura de leer la información nutricional de los alimentos y enseñar a realizarlo correctamente para así seleccionar los más adecuados.

Con respecto a los ejes transversales se dice que, aunque se crean para aplicarse en los centros educativos, no existe una reglamentación para la aplicación de estos. En el programa no existen lecciones designadas para implementar estos ejes transversales y la mayoría de docentes no los aplica, solamente los incluye en los planeamientos por si llegan a observarles, o si el director o directora lo pide como un requisito. En la Figura 4 el profesorado opinó en relación con la idea anterior.

Los datos anteriores indican que un $68 \%$ de docentes opina que en la mayoría de los casos los ejes transversales se aplicaron de forma inadecuada. Esto podría deberse a que no se cuenta con el tiempo necesario para abordar los temas o porque el profesorado no les está dando la importancia que se merecen, un acto educativo se base principalmente en el contenido disciplinar conceptual, el cual le ocupa y le preocupa todo el tiempo, dejando de lado el trabajo de las competencias del pensamiento científico, las cuales serán la clave para que el 
doi: http://dx.doi.org/10.15359/ree.21-3.12

URL: http://www.una.ac.cr/educare

CORREO: educare@una.cr

estudiantado pueda enfrentar su diario vivir y aportar a la sociedad soluciones adecuadas ante situaciones desconocidas o problemáticas.

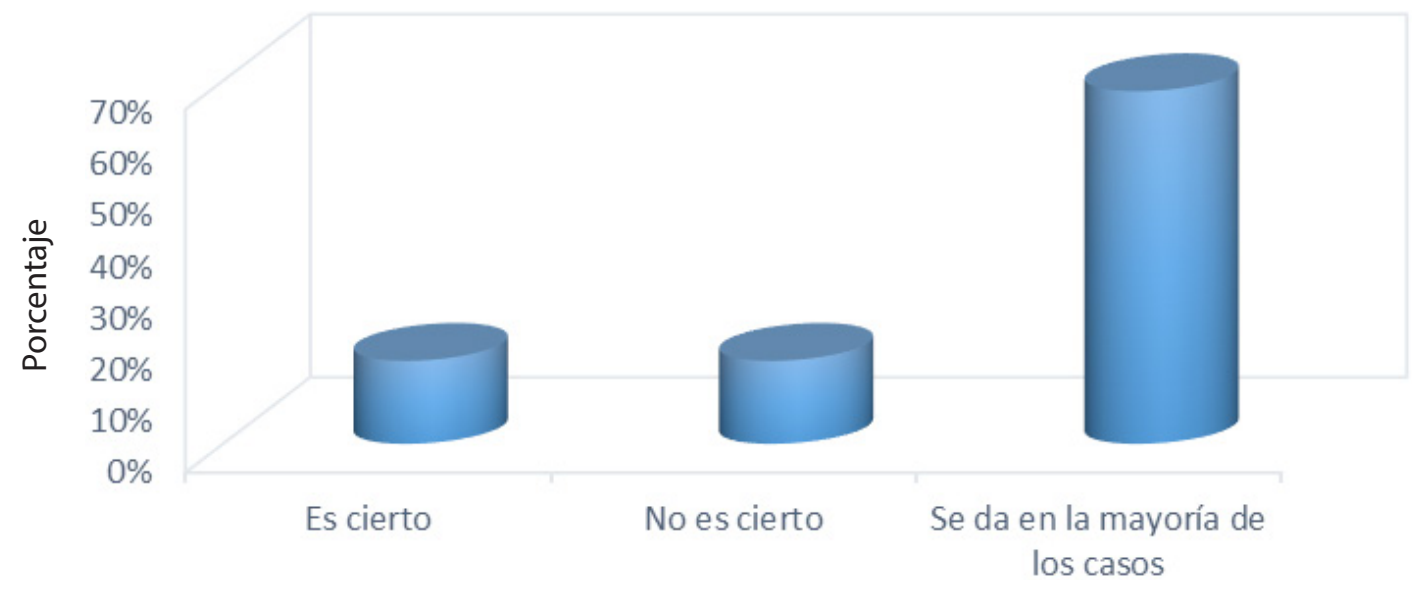

Opinión del profesorado

Figura 4: Frecuencia con la que docentes manifiestan la aplicación inadecuada de los ejes transversales.

A su vez, refleja que el profesorado no es persistente en la incorporación de los temas transversales; esto nos hace reflexionar sobre la necesidad de solicitar asesoramientos al MEP sobre la incorporación de la transversalidad en el currículo costarricense y que este se aborde desde la disciplina como elemento valorativo y formativa tanto del cuerpo docente como estudiantil de la institución.

La Figura 5 presenta las respuestas a la pregunta si en el proyecto educativo del colegio existen orientaciones que permitan incorporar la educación para la salud.

En la Figura 5 se muestra que un $66 \%$ no sabe si la institución realizó actividades para desarrollar el tema de nutrición, esto podría deberse a que la mayoría de docentes de la institución estudiada trabaja también en otros colegios para completar las lecciones y además viven lejos de la institución. Aun así, la responsabilidad de la aplicación del eje transversal, educación para la salud, es responsabilidad directa del personal docente y debe ser supervisada por la institución. Se recomienda al colegio realizar actividades innovadoras para desarrollar el tema de nutrición para potenciar los conocimientos y la motivación de sus estudiantes y así promover una cultura educativa responsable, analítica y reflexiva en torno a los buenos hábitos alimenticios. 


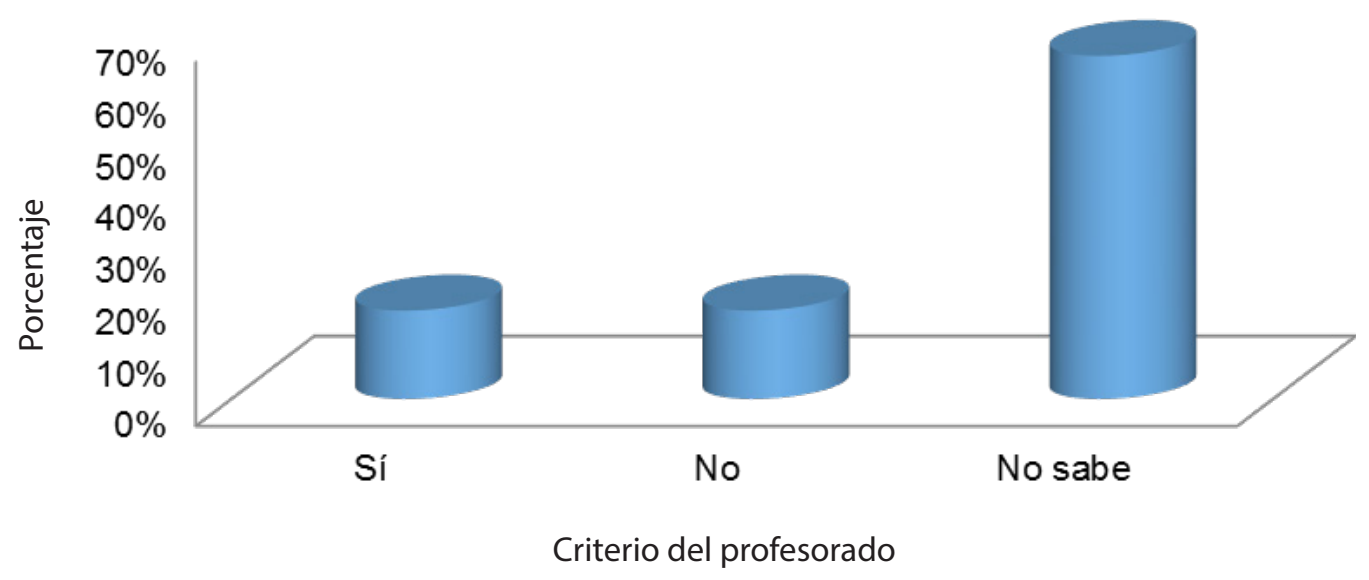

Figura 5: Criterio obtenido de docentes sobre la incorporación de la educación para la salud.

También se analizó si se recibió formación específica para desarrollar los temas transversales en su asignatura o especialidad. Del total de los 18 docentes de la entrevista, nadie ha recibido una formación específica para desarrollar los temas transversales en su materia, lo cual nos hace pensar en la necesidad de no solo sugerir la capacitación docente en cuanto a la incorporación y aplicación de los ejes transversales dentro del currículo, sino que también se deben generar mecanismos de presión para que se convierta en una realidad educativa y así se formule un plan nacional de capacitación relacionada con la disciplina y su transversalidad.

Finalmente, se obtuvo que un $22 \%$ de docentes considera que es inexistente la educación para la salud de su institución, se reflejó que no todo el personal educativo está dando énfasis a estas prácticas saludables que conllevan a una buena calidad de vida; además, los porcentajes correspondientes a insuficiente es de 78\%, valor que debería ser motivo de preocupación, ya que las repercusiones de una inadecuada educación nutricional pueden acarrear una serie de problemas en la salud aún peores que el desconocimiento. Esta situación a mediano plazo se podría plasmar en consecuencias sociales, económicas y familiares producto del tratamiento que los individuos deben recibir para minimizar sus conductas negativas ante el trastorno nutricional.

\section{Consideraciones finales}

Se evidencia la falta de información de docentes en relación con los buenos hábitos alimenticios y su efecto sobre la salud. También se refleja la necesidad de implementar estrategias efectivas que contribuyan a enraizar en la juventud hábitos alimenticios y de nutrición más sana, acordes con los postulados de las ciencias de la nutrición. Esto ocurre debido a que la enseñanza sobre el tema, en cursos anteriores, había sido superficial y desligada del verdadero 
doi: http://dx.doi.org/10.15359/ree.21-3.12

URL: http://www.una.ac.cr/educare

CORREO: educare@una.cr

contexto en el que se desenvuelve el estudiantado. Tal es el nivel de afectación que los sujetos encuestados tenían nociones sobre el tema, pero no se permeó en ellos, y algunos ni siquiera recuerdan si se les habló de ello.

Esta investigación no se aleja de la problemática universal que ha situado a la obesidad a la cabeza de muchos padecimientos inherentes a una población joven. Compete a docentes de todas las materias, en especial en ciencias experimentales, encausar al estudiantado hacia aprendizajes objetivos, significativos y coherentes en esta área en particular. La educación para la salud promueve la adquisición de conocimientos para desarrollar destrezas y prácticas de estilos de vida saludables hacia la prevención de enfermedades.

Una cantidad significativa de estudiantes compra comida chatarra fuera de las instituciones educativas, no consume merienda saludable elaborada en el hogar ni fruta fresca, lo cual podría ser indicador de que, desde el hogar, no se fomentan estilos de vida saludables.

En general el personal docente conoce que se debe consumir una dieta balanceada, que se deben comer frutas; pero no se lleva a la práctica en sus hábitos diarios de alimentación.

A pesar de estar incluido el tema en los contenidos transversales de los Programas de Estudio de Tercer Ciclo, la gran mayoría del profesorado no los ha interiorizado como una temática capital en el contexto histórico que vive el país y, en consecuencia, no se promueve en las lecciones cotidianas con el énfasis que merece esta problemática. Por lo tanto, es urgente que el personal docente plantee cambios en las prácticas didácticas tendientes a propiciar la motivación y la participación de sus estudiantes y, por otro lado, que la institución y el MEP sean vigilantes y promotores de que sus docentes promuevan los temas transversales como elemento importante en las competencias del pensamiento científico en el estudiantado.

\section{Referencias}

Aguirre, M. L., Castillo, C. y Le Roy, C. (2010). Desafíos emergentes en la nutrición del adolescente. Revista Chilena Pediatría, 81(6), 488-497. Recuperado de http://www.scielo.cl/pdf/rcp/ v81n6/art02.pdf

Albero, R., Sanz, A. y Playán, J. (2004). Metabolismo en el ayuno. Endocrinol Nutri, 51(4), 139-148. doi: https://doi.org/10.1016/S1575-0922(04)74599-4

Albito,T.M. (2015). Hábitos alimenticios y su influencia en el estado nutricional de los adolescentes del bachillerato del colegio Diez de Noviembre, de la Parroquia Los Encuentros, en el año 2014 (Tesis de licenciatura). Universidad Nacional de Loja, Sede Zamora. Zamora, Ecuador. Recuperado de http://dspace.unl.edu.ec/jspui/bitstream/123456789/9197/1/Teresa\%20 Margarita\%20Albito\%20Carrillo.pdf 
Calañas-Continente, A. J. (2005). Alimentación saludable basada en la evidencia. Endocrinología y Nutrición,52(Supl2),8-24.Recuperadodehttp://www.elsevier.es/es-revista-endocrinologianutricion-12-articulo-alimentacion-saludable-basada-evidencia-13088200

Castañeda-Sánchez, O., Rocha-Díaz, J. C., Ramos-Aispuro, M. G. (2008). Evaluación de los hábitos alimenticios y estado nutricional en adolescentes de Sonora, México. Archivos en Medicina Familiar, 10(1) 7-11. Recuperado de http://www.redalyc.org/articulo.oa?id=50713090003

Castillo, C.y Romo, M. (2006). Las golosinas en la alimentación infantil. Revista chilena de pediatría, 77(2). doi: https://doi.org/10.4067/S0370-41062006000200011

Daza, S. y Arrieta, J. R. (2010). La nutrición en los vegetales: Una unidad didáctica para la enseñanza de la biología (Cap. 1). En M. Quintanilla, S. Daza y C. Merino (Eds.), Unidades didácticas en biologíayeducaciónambiental.Sucontribuciónalapromocióndecompetenciasdepensamiento científico (Vol. 4, pp. 12-32). Santander, Colombia: GRECl. Recuperado de http://www7.uc.cl/ sw educ/educacion/grecia/plano/html/pdfs/destacados/LibroDBioGrecia11julio.pdf

Díaz, E. y Ramos, R. R. (2007). Transversalidad curricular para la temática familia en la carrera de medicina. Educación Medica Superior, 21(4), 1-11. Recuperado de http://scielo.sld.cu/pdf/ ems/v21n4/ems09407.pdf

FAO. (2011). La importancia de la educación nutricional. Roma: Autor. Recuperado de http://www. fao.org/ag/humannutrition/31779-02a54ce633a9507824a8e1165d4ae1d92.pdf

Fernández, A. (2003). Importancia de la nutrición en el atleta de tercera generación-veterano. Efdeportes.com, 8(58). Recupeado de http://www.efdeportes.com/efd58/nutri.htm

Flegal, K. M., Carroll, M. D., Ogden, C. L., \& Johnson, C. L. (2002). Prevalence and trends in obesity among US adults, 1999-2000. JAMA, 288(14), 1723-1727. doi: https://doi.org/10.1001/ jama.288.14.1723

French, S. A., Story, M., Fulkerson, J. A., \& Gerlach, A. F. (2003). Food environment in secondary schools: À la carte, vending machines, and food policies and practices. American Journal of Public Health, 93(7), 1161-1168. Recuperado de https://www.ncbi.nlm.nih.gov/pmc/ articles/PMC1447927/

Gómez, O. (2004). Educación para la salud. San José, Costa Rica: EUNED.

González, E y Merino, B. (Coord.). (2007). Alimentación saludable: Guía para las familias. España: Secretaría General Técnica del MEC. Recuperado de https://www.msssi. gob.es/profesionales/saludPublica/prevPromocion/promocion/saludJovenes/docs/ alimentSaludGuiaFamilias 2007.pdf 
doi: http://dx.doi.org/10.15359/ree.21-3.12

URL: http://www.una.ac.cr/educare

CORREO: educare@una.cr

Hernández, R., Fernández, C. y Baptista, M. (2010). Metodología de la investigación (5a ed.). México: McGraw-Hill Interamericana Editores.

Lujan, A. (2013). Incidencia de la cultura alimentaria en hábitos alimentarios de niños de 7 a 9 años de Bombal (Tesis de licenciatura). Universidad Abierta Interamericana, Rosario, Argentina. Recuperado de http://imgbiblio.vaneduc.edu.ar/fulltext/files/TC112603.pdf

Macias, A. I., Gordillo, L. G. y Camacho, E. J. (2012). Hábitos alimentarios de niños en edad escolar y el papel de la educación para la salud. Revista Chilena Nutrición, 39(3), 40-43. Recuperado de http://www.scielo.cl/pdf/rchnut/v39n3/art06.pdf

Marugán, J. M., Monasterio, L. y Pavón M. (2010). Alimentación en el adolescente. En SEGHNP (Ed.), Protocolos diagnóstico-terapéuticos de gastroenterología, hepatología y nutrición pediátrica SEGHNP-AEP (pp. 307-312). Madrid: ERGON. Recuperado de https://www. gastroinf.es/sites/default/files/files/Protocolos\%20SEGHNP.pdf

Méndez, J. (2012). Diseño curricular bajo el modelo de educación basada en normas por competencia. San José, Costa Rica: MEP. Recuperado de https://www.gastroinf.es/sites/ default/files/files/Protocolos\%20SEGHNP.pdf

Ministerio de Educación Pública, Costa Rica (MEP). (2012). Programa de estudio de ciencias. Tercer ciclo de educación general básica. San José, Costa Rica: Autor. Recuperado de http://cse. go.cr/sites/default/files/acuerdos/ciencias iii ciclo.pdf

Montero, A., Úbeda, N. y García A. (2006). Evaluación de los hábitos alimentarios de una población de estudiantes universitarios en relación con sus conocimientos nutricionales. Nutrición Hospitalaria, 21(4), 466-473. Recuperado de http://scielo.isciii.es/pdf/nh/v21n4/ original1.pdf

Neimeier, H. M., Raynor H. A., Lloyd-Richardson, E. E., Rogers, M. L., \& Wing, R. R. (2006). Fast food consumption and breakfast skipping: Predictors of weight gain from adolescence to adulthood in a nationally representative sample. The Journal of Adolescent Health, 39(6), 842-900. doi: https://doi.org/10.1016/j.jadohealth.2006.07.001

Palos, J. (2001). Transversalidad y temas transversales: Reinterpretar y cambiar el currículo. En OEI (Ed.), Memorias La educación en valores en Iberoamérica. Foro iberoamericano sobre educación en valores (pp. 37-52). Madrid: OEl. Recuperado de http://www.educativo.utalca. $\mathrm{cl} /$ medios/educativo/bliblioteca profesor/educacion valores.pdf

Real Academia Española (RAE). (2014). Diccionario de la lengua española (23 ed.). Recuperado de http://dle.rae.es/?w=diccionario

Restrepo, S. L., Morales R. M., Ramírez, M. C., López, M. V. y Varela, L. E. (2006). Los hábitos 
doi: http://dx.doi.org/10.15359/ree.21-3.12

URL: http://www.una.ac.cr/educare

alimentarios en el adulto mayor y su relación con los procesos protectores y deteriorantes en salud. Revista Chilena de Nutrición, 33(3), 58-62. Recuperado de http://www.redalyc. org/articulo.oa?id=46914636006

Sedó, P. (2009). Guía práctica para una adecuada alimentación de la persona adulta mayor y su familia ( $2^{\mathrm{a}}$ ed.). San José, Costa Rica: SIEDIN.

Segura, M. (2006). Los valores en el planeamiento didáctico. Eje transversal del currículo costarricense. San José, Costa Rica: MEP. Recuperado de https://es.slideshare.net/eclimosis/ valoresyplaneamiento-costa-rica-50643514

Serrú, L., Laclé, A. y Coto, C. (2003). ¿Sobrepeso o "achicamiento" en escolares de sexto grado en un área urbano marginal del área metropolitana. Revista Costarricense de Salud Pública, 12(22), 53-66. Recuperado de http://www.scielo.sa.cr/scielo.php?script=sci arttext\&pid $=\mathrm{S} 1409-14292003000200006$

Soriano, A. M. (2014). Diseño y validación de instrumentos de medición. Diá-logos, 14, 19-40. Recuperado de http://www.lamjol.info/index.php/DIALOGOS/article/view/2202/1997

Vilaplana, M. (2011). Educación nutricional en el niño y el adolescente. Objetivos clave. Offarm, 30(3), 43-50. Recuperado de http://www.elsevier.es/es-revista-offarm-4-articuloeducacion-nutricional-el-nino-adolescente--X0212047X11205090 